\section{A randomized controlled trial of an enhanced interdisciplinary community based group program for people with Parkinson's disease: study rationale and protocol}

\author{
Catherine Peters, ${ }^{1,2}$ Michelle Currin, 1 \\ Sara Tyson, 1 Anthea Rogers, 1 \\ Susan Healy, 1 Steven McPhail,3,4 \\ Sandra G. Brauer, 5 \\ Katharine Heathcote,2,6 Tracy Comans 2,7 \\ ${ }^{1}$ Community Rehabilitation Service, Metro \\ South Health Service District; ${ }^{2 S c h o o l ~ o f ~}$ \\ Medicine, Griffith University; ${ }^{3}$ Centre for \\ Functioning and Health Research, \\ Queensland Health; ${ }^{4}$ School of Public \\ Health and Institute Health of Biomedical \\ Innovation, Queensland University of \\ Technology; 5 School of Health and \\ Rehabilitation Sciences, The University of \\ Queensland; 6University Centre for Rural \\ Health (North Coast), University of \\ Sydney; ${ }^{7}$ Griffith Health Institute, \\ Griffith University, Australia
}

\section{Abstract}

Parkinson's disease (PD) is a progressive, chronic neurodegenerative disorder for which there is no known cure. Physical exercise programs may be used to assist with the physical management of PD. Several studies have demonstrated that community based physical therapy programs are effective in reducing physical aspects of disability among people with PD. While multidisciplinary therapy interventions may have the potential to reduce disability and improve the quality of life of people with PD, there is very limited clinical trial evidence to support or refute the use of a community based multidisciplinary or interdisciplinary programs for people with PD. A two group randomized trial is being undertaken within a community rehabilitation service in Brisbane, Australia. Community dwelling adults with a diagnosis of Idiopathic Parkinson's disease are being recruited. Eligible participants are randomly allocated to a standard exercise rehabilitation group program or an intervention group which incorporates physical, cognitive and speech activities in a multi-tasking framework. Outcomes will be measured at 6 -week intervals for a period of six months. Primary outcome measures are the Montreal Cognitive Assessment (MoCA) and the Timed Up and Go (TUG) cognitive test. Secondary outcomes include changes in health related quality of life, communication, social participation, mobility, strength and balance, and carer burden measures. This study will determine the immediate and long-term effectiveness of a unique multifocal, interdisciplinary, dual-tasking approach to the management of PD as compared to an exercise only program. We anticipate that the results of this study will have implications for the development of cost effective evidence based best practice for the treatment of people with PD living in the community.

\section{Introduction}

Parkinson's disease (PD) is a progressive, neurological disorder and is one of the most common chronic neurodegenerative diseases in the elderly population. While the main treatment for PD is pharmacological management through dopamine substitution therapy, this does not improve all symptoms. ${ }^{1}$ Many people with PD have a wide range of rehabilitation needs due to difficulties with balance and mobility, activities of daily living, cognition, speech and swallow, depression, fatigue, sleep disorders and continence that are not controlled through medication. Despite optimal pharmacological treatment, motor function such as gait, posture, balance and speech progressively deteriorate impairing mobility, self care, communication and participation. ${ }^{2}$

In the community setting, rehabilitation programs for individuals with PD have been primarily aimed at improving motor function. Physiotherapy exercise programs have been shown to improve physical outcomes, including gait performance, ${ }^{3}$ balance ${ }^{3-5}$ and strength $^{5,6}$ in people with $\mathrm{PD}$, highlighting the importance of exercise in these individuals. However, with advancing age and disease duration, there is an increased risk of cognitive and neuropsychiatric decline. ${ }^{7}$ Non-motor complications, such as cognitive impairment and depression worsen the prognosis of PD and increase the personal and socioeconomic burden of this disease. ${ }^{8}$ However, these concerns are less commonly addressed by community services.

Cognitive dysfunction in those with PD is common. The prevalence of mild cognitive defects among people with PD without dementia, has been reported to be between 24 to $55 \%$, even in the early stages of the disease, $, 9,10$ with those newly diagnosed being twice as likely to develop mild cognitive impairment than healthy elderly.9,11 Executive function, attention, memory, visuospatial dysfunction and psychomotor speed are aspects of cognition most affected by PD. ${ }^{10,12}$ Cognitive deficits of this nature have the potential to impact almost
Correspondence: Catherine Peters, Community Rehabilitation Service, Metro South Health Service District, P0 Box 4069, Eight Mile Plains QLD 4113, Australia.

Tel. +61.7.3169.9747 - Fax: +61.7.3169.9701.

E-mail: catherine_peters@health.qld.gov.au

Key words: Parkinson's disease, rehabilitation, clinical protocol, allied health.

Acknowledgments: this study has been funded by The Griffith University Primary Health Care Research Evaluation and Development Program, funded by the Australian Government Department of Health and Ageing; The Health Practitioner Research Grants Scheme, Queensland Health; Occupational Therapists Board of Queensland and Occupational Therapy Australia Qld Clinical Research Bursary. Trial Registration: The protocol for this study is registered with the Australian and New Zealand Clinical Trial Registry (Registration n. ACTRN12610000716066).

Received for publication: 1 November 2011. Accepted for publication: 28 December 2011.

This work is licensed under a Creative Commons Attribution NonCommercial 3.0 License (CC BYNC 3.0).

(C) Copyright C. Peters et al., 2012

Licensee PAGEPress srl, Italy

Neurology International 2012; 4:e3

doi:10.4081/ni.2012.e3

all aspects of life and compound the physical dysfunction experienced by those with PD. Management of cognitive impairment in PD has been limited in current clinical guidelines to the use of pharmacological interventions such as cholinesterase inhibitors which have been shown to have a small benefit. ${ }^{13}$ A preliminary study of a small group of people with PD demonstrated that cognitive training that targeted attention, abstract reasoning and visuospatial skills improved aspects of cognition reliant on frontal lobe function compared to baseline results. ${ }^{14}$ Results from this study indicated lasting improvements in verbal fluency and recall over a 6-month period, emphasising the importance of continued mental stimulation in the preservation of cognitive capacity.

It is estimated that $89 \%$ of people with PD have speech and voice disorders ${ }^{15}$ with the most common being deficits in prosody, phonation and articulation. ${ }^{16}$ These deficits can have a considerable impact on the social and emotional wellbeing of people with PD. The reduced ability to communicate is considered to be one of the most difficult aspects of PD for many individuals with this disease and their families. ${ }^{17}$ Intensive individual speech training, including the Lee Silverman Voice Treatment (LSVT ${ }^{\circledR}$ LOUD), has been shown to 
improve speech deficits. ${ }^{18,19}$

These programs described in the literature train speech and cognitive tasks in isolation. However, these tasks are usually performed in conjunction with physical activities during normal activities of daily living. For example, walking and talking or following a recipe while standing and cooking. These dual-tasks have been shown to be particularly adversely impacted by PD. ${ }^{20-22}$ Previous programs have focused on limited aspects of these tasks, and have not integrated both speech and cognitive elements into an exercise program.

Allied health interventions for people with PD are typically conducted in isolation despite many overlapping treatment strategies and complementary goals. ${ }^{1}$ Due to the complexity and array of problems faced by these individuals, combining therapies can potentially promote an integrated approach to these elements of function (e.g. speech, cognition, motor function), and improve outcomes for these patients, compared to individual therapy interventions that are delivered in isolation. The effectiveness of integrated programs in PD have not been reported. However, positive outcomes of integrated care programs in other chronic conditions have been identified. ${ }^{23}$

A systematic review of rehabilitation outcomes in PD24 identified 44 studies, 43 of which were single discipline rehabilitation interventions. Another recent systematic review 25 similarly reported insufficient of high level evidence to demonstrate whether multidisciplinary outpatient programs produce effective short or longer term outcomes for people with PD. In addition, no studies have been identified that examine whether delivering an interdisciplinary PD specific program with dual-tasking activities improves not only physical abilities but also the quality of life and the cognition of PD clients.

The primary aim of this randomized controlled trial is to evaluate the effectiveness of an interdisciplinary, multifactorial group program that incorporates physical, cognitive and speech dual-task activities on cognition and physical function compared with a standard exercise rehabilitation program. In addition, longer term outcomes will also be assessed to determine the carryover effects of both programs at intervals up to six months to evaluate what is the optimum time to offer a maintenance program to these people.

\section{Materials and Methods}

\section{Study design}

This study is a randomized controlled trial with concealed allocation and blinded assessment of measures repeated at 6 -week intervals for a period of six months and will be analyzed with intention-to-treat analysis. The control group will receive a standard exercise group rehabilitation program and the intervention group will receive the enhanced program that includes the standard exercise group rehabilitation program and 'dual-task' cognitive and speech activities.

This trial has been designed according to CONSORT guidelines. The study protocol has been approved by the Princess Alexandra Hospital Human Research Ethics Committee and the Griffith University Research Ethics Committee.

\section{Recruitment procedure}

Neurologists within the Brisbane Metropolitan area will be contacted by mail and invited to refer to the study those adults with PD who live within the catchment area and meet the following eligibility criteria: i) confirmed diagnosis of idiopathic PD using the UK Brain Bank criteria; ii) independently mobile (with or without mobility aid); iii) living at home in the community; iv) rated stage I-III on the Hoehn and Yahr disability scale; ${ }^{26}$ v) medical approval to participate in a moderate-intensity exercise program. Clients will be excluded if: they i) live in a residential care facility (low or high level care); or ii) have a cognitive or physical impairment that affects their ability to participate in a community based program.

Following receipt of a referral, those referred will be contacted to discuss the project and arrange an initial home visit at which time informed consent for participation in the study will be obtained. The home visit will be conducted by an occupational therapist and speech pathologist or physiotherapist. An initial assessment will be conducted to assess the person with current PD functional status, including level of independence in activities of daily living, physical (balance, mobility, level of physical activity) and cognitive status, to determine if they are appropriate for inclusion in the study, i.e. able to complete a moderate intensity exercise program and complete assessment tools. Immediate individual therapy needs are addressed at this time. This may include prescription of mobility devices, home environment audit or referral to other relevant services.

Those who are eligible and who consent will undertake further baseline assessment including the Unified Parkinson's Disease Rating Scale (MDS-UPDRS), ${ }^{27}$ motor subsection, ${ }^{28}$ Montreal Cognitive Assessment (MoCA), and the Parkinson's Disease Questionnaire (PDQ39). ${ }^{29}$ All study participants will be asked to refrain from initiating any other new exercise programs or other allied health therapy intervention during their participation in the study. Those excluded or who do not agree to participate in the research study will be offered other appropriate therapy intervention (e.g. homebased exercise program) or referred to other relevant agencies.

\section{Randomization}

Following baseline assessment, participants will be formally entered into the study and randomized to either the intervention (interdisciplinary) or control (exercise therapy) groups. Randomization will be performed according to a random list of numbers generated by computer and undertaken in blocks of 8-12 with no more than 6 participants allocated to each group. The allocation list will be handled by an independent investigator who will have no contact with the study participants while undertaking the program and will not be involved in the supervision of staff responsible for data collection.

\section{Treatment programs}

Both programs will consist of two 1.5-2 $\mathrm{h}$ group sessions per week over a 4-week period, progressively graded in complexity over this time. The group sessions will be performed by a physiotherapist, therapy assistant, and an occupational therapist or speech pathologist in a community health center. A maximum of 6 participants will be allocated to each group. The two groups will be exposed to the same length of intervention, social interaction and contact with the program facilitators. All sessions will be delivered in a similar structured format for consistency and will be progressive.

\section{Standard group exercise program (control and intervention group)}

The standard exercise program is based on evidence-based guidelines for physiotherapy in clinical practice.30-32 All sessions will commence with a 5 min Tai Chi warm up and 15 min of a Tai Chi sequence. The 6 -form Tai Chi sequence program involves movement components such as body and trunk rotation, flexion and extension of hips and knees, weight shifting, coordinated arm movements, and postural alignment and control. ${ }^{33,34}$ Due to the combination of deep breathing and relaxation with slow and gentle movements, Tai Chi has been found to be amenable to PD patients with posture-related instability problems. ${ }^{34}$ This will be followed by a $40 \mathrm{~min}$ exercise circuit. The exercise circuit is designed to address areas of reduced physical function that are commonly experienced by those with PD, such as deficits in standing balance, postural alignment, gait disturbances, rigidity, bradykinesia, reduction in movement range, decline in strength (upper limb and lower limb), and reduced cardiovascular fitness.

Balance activities include altering the base of support incorporated with varying surfaces and graded tasks reaching outside base of sup- 
port while standing. Gait practice will include visual and auditory cueing techniques to improve step length, velocity, amplitude, arm swing and cadence, practice of initiation and termination, and dual-tasking activities whilst walking and negotiating different obstacles. ${ }^{35}$ Functional strengthening activities for lower limb muscle groups include sit-to-stand, step activities, heel-raise in standing and minisquat exercises. Upper limb strengthening activities utilize free weights and graded resistance bands. Endurance activities include exercise bike, pedal and rowing machine, increasing demand through increased distance and/or resistance. Throughout all activities, postural awareness is promoted. All participants are provided with a home exercise program which includes a range of balance, postural and strengthening exercises that have been demonstrated in the program.

\section{Enhanced program \\ (intervention group)}

The enhanced program includes all aspects of the standard (exercise) program as outlined above, as well as cognitive and speech components. During the first week of the program, cognitive and speech components are conducted separately from the physical component of the program. These two sessions are conducted with the client seated in a group meeting room. Cognitive and speech concepts are introduced and relevant strategies are discussed and practiced. Topics covered include attention, memory, visual perception and factors influencing speech clarity. Participants are provided with handouts that reinforce cognitive and speech strategies that are discussed. For the following three weeks, cognitive and speech work stations are incorporated into the exercise circuit in addition to the standard exercise program and performed in the gym.

The cognitive work station includes activities that focus on executive functioning, atten- tion, memory and visuospatial activities. Cognitive activities are undertaken in pairs and assistance provided by clinicians as required. Cognitive strategies taught in previous sessions are practised and reinforced throughout these tasks. The activities are graded over the eight sessions and include Sudoku, number wheels, visual perceptual scanning in pictures, mathematical calculations, and word-based activities such as 20 questions, hangman, find-a-word, word ladders and scrambled words. The activities that are utilized in the program have been chosen because they are age appropriate, readily accessible, and facilitate social interaction.

The speech work station includes activities targeting the deficits in phonation, articulation and prosody. Tasks aim to practise the behavioral strategies introduced and discussed in week one, including use of increased breath support for speech, increased articulatory and vocal effort, and monitoring of speech output and the responses of listeners. The speech tasks are graded across the sessions and include the reading of everyday sentences (week 2), sentences of increasing complexity, tongue twisters, prosody drills (week 3), limericks and simple dialogues (week 4). Tasks are undertaken in pairs and participants are encouraged to evaluate and give feedback to their partner regarding the clarity of their speech output. Each task is initially completed while standing, with performance demands gradually being increased by the addition of distance between the speaker and listener and the subsequent requirement for walking while talking.

A further important component of the enhanced program is the graded inclusion and integration of cognitive, speech and physical demands on participants while they complete various workstations. For example, clients will initially perform a task such as a Sudoku sitting down with assistance from a staff member (week 2). This will be graded through to having to do the task while standing and while discussing the responses with another participant using clear verbal responses (week 3), to having to write the numbers on the Sudoku which has been positioned at head height on a wall while clearly conversing with other study participants and therapy staff. This integrated approach enables the cognitive, speech and physical goals of the three allied health disciplines utilized in this program to be addressed.

A half-hour self-management component is included in each session of the enhanced program. This component of the program is based on self-management principles and aims to increase the clients' knowledge of their condition and provide them with strategies to help them maintain their independence within their community. Group discussion is a key component of these sessions. Cognitive and speech strategies are reinforced as part of the management strategies discussed in these sessions (Table 1).

\section{Measuring outcome}

The primary outcomes are concerned with measuring cognition and physical function with cognitive demand. The Montreal Cognitive Assessment (MoCA) ${ }^{28}$ is a brief screening tool that is reportedly sensitive to detecting mild cognitive impairment in patients with PD. 36,37 The Timed Up and Go (TUG) cognitive 38 instrument measures the effect of cognitive demands on functional mobility. It involves adding a cognitive task (subtracting 7 from 100) while performing the TUG test, the time taken to rise from a chair, walk $3 \mathrm{~m}$, turn around, walk back and sit down using their usual gait aid.

Secondary outcome measures include: health related quality of life, physical measures for mobility, motor function, balance and upper limb dexterity, communication, depression, social participation and carer burden.

Table 1. Enhanced (intervention) and standard (control) group therapy programs.

\begin{tabular}{|c|c|c|}
\hline Module & Enhanced program & Standard program \\
\hline Exercise & $\begin{array}{ll}\text { Workstations of task-specific strategies: } \\
\text { - Gait } & \text { - Turning / rotation } \\
\text { - Balance } & \text { - Range of motion } \\
\text { - Cardiovascular } & \text { - Tai Chi } \\
\text { - Strengthening } & \end{array}$ & $\begin{array}{ll}\text { Workstations of task-specific strategies : } \\
\text { - Gait } & \text { - Turning / rotation } \\
\text { - Balance } & \text { - Range of motion } \\
\text { - Cardiovascular } & \text { - Tai Chi } \\
\text { - Strengthening } & \end{array}$ \\
\hline Speech & $\begin{array}{l}\text { Activities that address: } \\
\text { - Breath support for speech } \\
\text { - Prosody } \\
\text { - Speech Clarity }\end{array}$ & Nil \\
\hline Cognition & $\begin{array}{l}\text { Activities that address: } \\
\text { - Executive function } \\
\text { - Attention } \\
\text { - Memory } \\
\text { - Visuospatial awareness }\end{array}$ & $\begin{array}{l}1 / 2 \mathrm{~h} \text { in total rest and informal chat between group members } \\
\text { built into physical group. }\end{array}$ \\
\hline Education & $\begin{array}{l}\text { Self-management education } \\
\text { including group discussion }\end{array}$ & \\
\hline
\end{tabular}


These outcome measures are commonly used in assessment of individuals with PD in clinical practice.

Additional information recorded and obtained from the referring neurologist and study participant will include: demographics (age, sex, living arrangements), clinical characteristics (medical history, disease duration, details of current medications), and previous allied health therapy interventions. Changes in medication or medical management will also be recorded at each assessment. All outcome measures are shown in Table 2.

\section{Testing}

Subjects will be tested the week before commencing the program at an assessment clinic at a community health center and one week after completing the program by research assistants (occupational therapists and physiotherapists) who are blinded to group allocation. Scripts for all measures are used during testing to ensure that all subjects receive the same instructions. A further three follow-up reassessments will take place at 6-week intervals commencing six weeks after the completion of the program and continuing until the 6-month time point (Figure 1) to determine the carryover effects of both programs. Assessments for each study participant are to be undertaken at the same time of day to minimize the effect of medication cycle dose fluctuations on results. Carers will be provided with relevant assessments to complete at these times. All assessments take between 60 to 90 min to complete.

\section{Statistical analysis \\ Sample size}

This trial aims to recruit 92 people with a diagnosis of ideopathic Parkinson's disease. For the TUG Cognitive, a minimal clinically important difference (MCID) of $3 \mathrm{sec}$ with a standard deviation of 3.6 was estimated from the report by Shumway-Cook and Brauer (38) and confirmation with the authors. This analysis calculated that a sample size of 23 persons per group would be required to detect a difference at $\mathrm{P}=+0.05$ with a power of 0.8 . Power calculations for the MoCA were based on MCID of 2 points given that this moves someone from mild cognitive impairment (measured as 25-26 points) to a normal range and a standard deviation of 3.1 based on previous values from a similar patients population. A sample size of 38 people per group would be required to detect a difference at $\mathrm{P}=0.05$ with a power of 0.8 . Factoring in an attrition rate of $20 \%$, it is proposed that 92 people (46 in each program) in total would be required to detect a difference in all primary outcome measures. The trial has commenced on a pilot basis with the aim of recruiting half the participants required

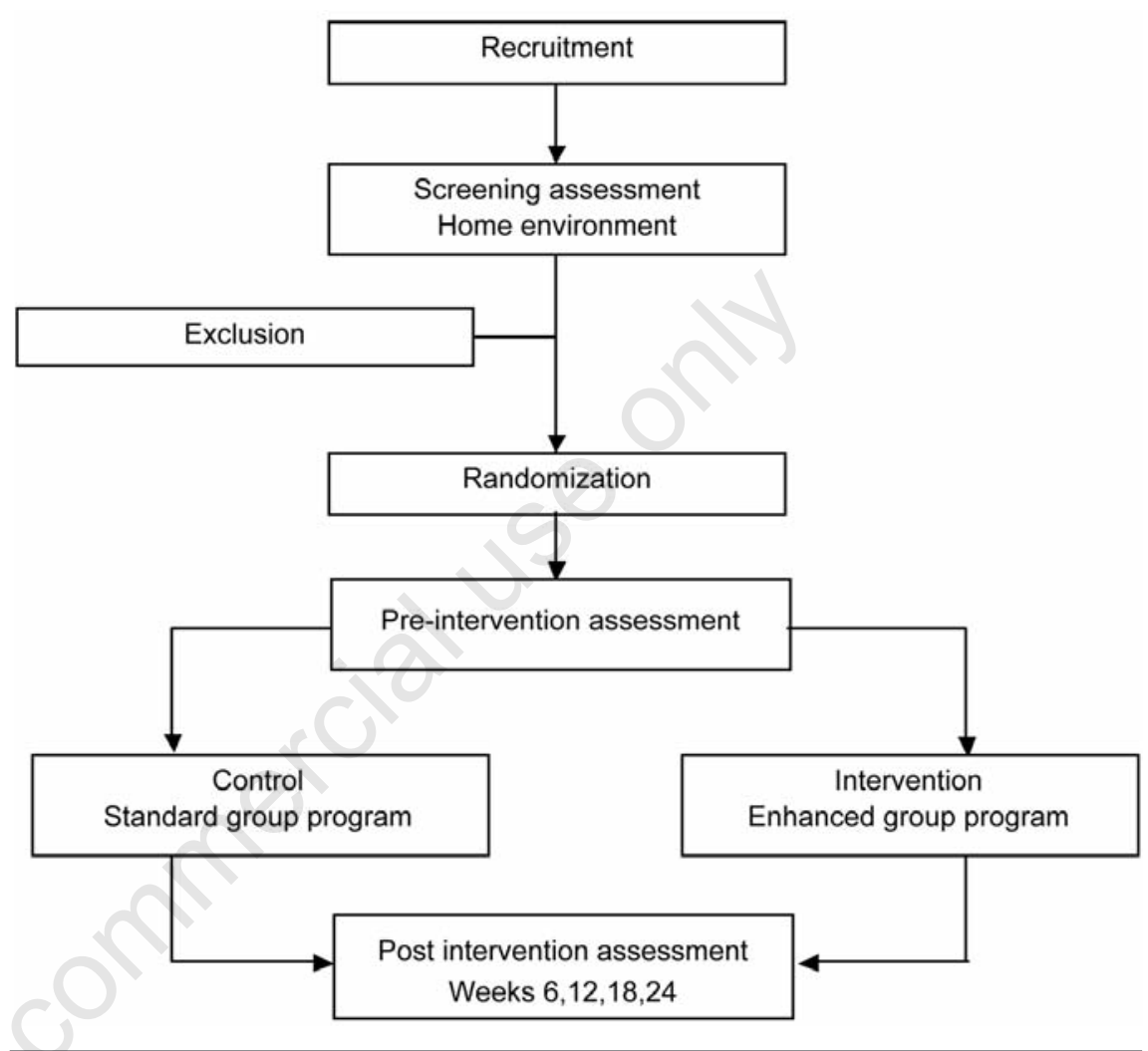

Figure 1. Trial design.

Table 2. Outcome measures.

\begin{tabular}{|c|c|c|}
\hline Construct & Descriptor & Measurement name \\
\hline Body structure and function & $\begin{array}{l}\text { Mental functions - cognition } \\
\text { Mental functions - depression } \\
\text { Muscle power }\end{array}$ & $\begin{array}{c}\text { Montreal Cognitive Assessment }{ }^{28 *} \\
\text { Geriatric depression scale }{ }^{40} \\
\text { Quads strength }{ }^{41}\end{array}$ \\
\hline Activity & $\begin{array}{c}\text { Mobility } \\
\text { Balance } \\
\text { Upper limb dexterity } \\
\text { Speech and language }\end{array}$ & $\begin{array}{c}\text { Timed Up and Go (TUG) }{ }^{42} \\
\text { Timed Up and Go cognitive (TUG Cognitive) })^{38 *} \\
10 \text { m test } 43 \\
\text { External perturbation }{ }^{44,45} \\
\text { Step test } 46,47 \\
\text { Nine Hole Peg Test } 48 \\
\text { Voice Handicap Index } 49 \\
\text { Speech clarity visual analogue scale } \\
\text { Speech clarity visual analogue scale - carer }\end{array}$ \\
\hline Participation & Global & Frenchay Activities Index ${ }^{50}$ \\
\hline Environmental factors & Support and relationship & $\begin{array}{c}\text { Carer strain index }{ }^{51} \\
\text { Carer Experience Scale }\end{array}$ \\
\hline Global & $\begin{array}{l}\text { Quality of life } \\
\text { Utility }\end{array}$ & $\begin{array}{c}\text { Parkinson's Disease Questionnaire (PDQ-39) }{ }^{29} \\
\text { Health Utilities Index (HUI3) } 53\end{array}$ \\
\hline
\end{tabular}

${ }^{*}$ Primary outcome measures. 
by the end of 2011 .

\section{Analysis of outcome measures}

Data will be analyzed according to intentionto-treat principles. Descriptive analytical methods will be used to examine the frequency distribution of the main variables, and mean measures, standard deviations and standard errors will be calculated for continuously distributed variables. Primary and secondary outcome measures that are continuous will be compared between groups and across time periods using a generalized estimating equation (GEE). The design of the study is longitudinal with repeated measurements which are correlated with another in an individual. The GEE is a flexible way of analyzing this type of design which takes into account the correlation between measures within an individual. Imputation of missing values will not be required as the GEE is able to handle missing data from individual participants over a time series. 39

\section{Discussion}

At present, limited specialized allied health services are available in the community for individuals with PD who have complex needs that are often not addressed by individual disciplines. An integrated multidisciplinary approach to the management of PD improves communication between disciplines, and enables shared goal setting and treatment plans that address the individual needs of the person with PD.

Speech and cognitive deficits are common in PD. While previous programs have been designed to address these individual deficits, ${ }^{14,18,19}$ such programs have not addressed the dual-tasking aspect of these problems that is a substantial burden to people with PD. This study is unique in that it combines and merges cognitive, speech and physical components into an interdisciplinary approach, and will rigorously measure the effect of this program on cognitive function, as well as physical outcomes both immediately following the program and for a follow-up period of six months.

Over time, due to the progression of PD and the often associated decline in motivation, the effects of rehabilitation programs diminish. As a result, individuals with PD may require some further intervention or support. It is not currently known when the effects of a community based rehabilitation program are lost and when re-intervention or top-ups may be required. This study will improve this knowledge gap, as it is designed to detect the rate of this decline over time by re-assessing participants on a 6 -week basis up to six months post program.
Results from this study will help clinicians and policy makers identify appropriate rehabilitation maintenance programs for people living in the community with PD, and will also identify what programs are best suited to help keep active participants functioning in their community. The outcomes from this research will contribute significantly to the evidence base regarding service provision for this client group. We also anticipate that it will guide the development of best practice guidelines within primary and community health care for this patient group and further inform clinical practice.

\section{References}

1. van der Marck MA, Kalf JG, Sturkenboom IHWM, et al. Multidisciplinary care for patients with Parkinson's disease. Parkinsonism \& Related Disorders 2009;15:S21923.

2. Ransmayr G. Physical, occupational, speech and swallowing therapies and physical exercise in Parkinson's disease. Journal of Neural Transmission 2011;118: 773-81.

3. Nieuwboer A, Kwakkel G, Rochester L, , et al. Cueing training in the home improves gait-related mobility in Parkinson's disease: the RESCUE trial. J Neurol Neurosurg Psychiatry 2007;78:134-40.

4. Ashburn A, Fazakarley L, Ballinger C, et al. A randomised controlled trial of a home based exercise programme to reduce the risk of falling among people with Parkinson's disease. J Neurol Neurosurg Psy-chiatry 2007;78:678-84.

5. Hirsch M, Toole T, Maitland C, Rider R. The effects of balance training and highintensity resistance training on persons with idiopathic Parkinson's disease. Arch Phys Med Rehabil 2003;84:1109-17.

6. Dibble L, Hale T, Marcus R, et al. Highintensity resistance training amplifies muscle hypertrophy and functional gains in persons with Parkinson's disease. Mov Disord 2006;21:1444-52.

7. Aarsland D, Andersen K, Larsen JP, et al. The Rate of Cognitive Decline in Parkinson Disease. Arch Neurol 2004;61:1906-11.

8. Riedel 0, Klotsche J, Spottke A, et al. Cognitive impairment in 873 patients with idiopathic Parkinson's disease. Results from the German Study on Epidemiology of Parkinson's disease with Dementia (GEPAD). J Neurol 2008;255:255-64.

9. Foltynie T, Brayne CEG, Robbins TW, Barker RA. The cognitive ability of an incident cohort of Parkinson's patients in the UK. The CamPaIGN study. Brain 2004;127: 550-60.

10. Muslimovic D, Post B, Speelman JD,
Schmand B. Cognitive profile of patients with newly diagnosed Parkinson disease. Neurology 2005;65:1239-45.

11. Aarsland D, Bronnick K, Larsen JP, et al. Cogntive impairment in incident, untreated Parkinson disease: the Norwegian ParkWest study. Neurology 2009;72:1121-6.

12. Caviness JN, Driver-Dunckley E, Connor DJ, et al. Defining mild cognitive impairment in Parkinson's disease. Movement Disorders 2007;22:1272-7.

13. 0'Brien JT, Burns A. Clinical practice with anti-dementia drugs: a revised (second) consensus statement from the British Association for Psychopharmacology J Psychopharmacol 2011;25:997-1019.

14. Sinforiani E, Banchieri L, Zucchella C, et al. Cognitive Rehabilitation in Parkinson's Disease. Archives of Gerontology and Geriatrics 2004;38:387-91.

15. Ramig L, Fox C, Sapir S. Speech treatment for Parkinson's disease. Expert Review of Neurotherapeutics 2008;8:297-303.

16. Darley FL, Aronson AE, Brown JR. Motor Speech Disorders. WB Saunders, Philadelphia, 1975.

17. Miller N, Noble E, Jones D, Burn D. Life with communication changes in Parkinson's disease. Age and Ageing 2006;35: 235-9.

18. Fox CM, Ramig LO, Ciucci MR, et al. The science and practice of LSVT/LOUD: neural plasticity-principled approach to treating individuals with Parkinson disease and other neurological disorders. . Semin Speech Lang 2006;27:283-99.

19. Sapir S, Spielman JL, Ramig LO, et al. Effects of Intensive Voice Treatment (the Lee Silverman Voice Treatment [LSVT]) on Vowel Articulation in Dysarthric Individuals with Idiopathic Parkinson Disease: Acoustic and Perceptual Findings. Journal of Speech, Language, and Hearing Research 2007;50:899-912.

20. Yogev G, Giladi N, Peretz C, et al. Dual tasking, gait rhythmicity, and Parkinson's disease: Which aspects of gait are attention demanding? Eur J Neurosci 2005;22: 1248-56.

21. Spildooren J, Vercruysse S, Desloovere K, et al. Freezing of gait in Parkinson's disease: The impact of dual-tasking and turning. Movement Disorders 25:2563-70.

22. Plotnik M, Dagan Y, Gurevich T, et al. Effects of cognitive function on gait and dual tasking abilities in patients with Parkinson's disease suffering from motor response fluctuations. Experimental Brain Research 208:169-79.

23. Ouwens M, Wollersheim H, Hermens R, et al. Integrated care programmes for chronically ill patients: a review of systematic reviews. International Journal for Quality in Health Care 2005;17:141-6. 
24. Gage H, Storey L. Rehabilitation for Parkinson's disease: a systematic review of available evidence. Clinical Rehabilitation 2004;18:463-82.

25. Johnston M, Chu E. Does attendance at a multidisciplinary outpatient rehabilitation program for people with Parkinson's disease produce quantitative short term or longer term improvements? A systematic review. NeuroRehabilitation 2010;26:37583.

26. Hoehn M, Yahr M. Parkinsonism: onset, progression and mortality. Neurology 1967;17:427.

27. Goetz CG, Tilley BC, Shaftman SR, et al. Movement Disorder Society-sponsored revision of the Unified Parkinson's Disease Rating Scale (MDS-UPDRS): Scale presentation and clinimetric testing results. Movement Disorders 2008;23: 2129-70.

28. Nasreddine ZS, Phillips NA, Bédirian V, et al. The Montreal Cognitive Assessment, MoCA: A Brief Screening Tool For Mild Cognitive Impairment. Journal of the American Geriatrics Society 2005;53:6959.

29. Jenkinson C, Fitzpatrick R, Peto V, et al. The Parkinson's Disease Questionnaire (PDQ-39): development and validation of a Parkinson's disease summary index score Age Ageing 1997;26:353-7.

30. Keus S, Hendriks H, Bloem B, et al. Clinical practice guidelines for physical therapy in patients with Parkinson's disease. Royal Dutch Society for Physical Therapy 2004;114.

31. Keus SHJ, Bloem BR, Hendriks EJM, Bredero-Cohen AB, Munneke M. Evidencebased analysis of physical therapy in Parkinson's disease with recommendations for practice and research. Movement Disorders. 2007;22(4):451-60.

32. Keus SHJ, Munneke M, Nijkrake MJ, Kwakkel G, Bloem BR. Physical therapy in Parkinson's disease: Evolution and future challenges. Movement Disorders. 2009;24 (1):1-14

33. Hackney M, Earhart G. Tai Chi improves balance and mobility in people with
Parkinson disease. Gait Posture 2008;28: 456-60.

34. Li F, Harmer P, Fisher J, et al. Tai ChiBased Exercise for Older Adults with Parkinson's Disease: A Pilot-Program Evaluation. Journal of Aging and Physical Activity 2007;15:139-51.

35. Brauer SG, Woolacott MH, Lamont R, et al. Single and dual task gait training in people with Parkinson's Disease: A protocol for a randomised controlled trial. BMC Neurology 2011;11.

36. Nazem S, Siderowf AD, Duda JE, et al. Montreal Cognitive Assessment Performance in Patients with Parkinson's Disease with "Normal" Global Cognition According to Mini-Mental State Examination Score. Journal of the American Geriatrics Society 2009;57:304-8.

37. Gill DJ, Freshman A, Blender JA, Ravina B. The Montreal cognitive assessment as a screening tool for cognitive impairment in Parkinson's disease. Movement Disorders 2008;23:1043-6

38. Shumway-Cook A, Brauer S, Woollacott M. Predicting the Probability for Falls in Community-Dwelling Older Adults Using the Timed Up \& Go Test. Phys Ther 2000;80:896-903.

39. Burton P. Extending the simple linear regression model to account for correlated responses: An introduction to generalized estimating equations and multi-level mixed modelling Statistics in Medicine 1998;17:1261-91.

40. Brink TL. Proper scoring of the geriatric depression scale. J Am Geriatr Soc 1989; 37:819-20.

41. Bohannon R, Shove M, Bareeca S, et al. Five-repetition sit-to-stand test performance in community-dwelling adults: A preliminary investigation of times, determinants, and relationship with self-reported physical performance. Isokinetics and Exercise Science 2007;15:77-81.

42. Podsiadlo D, Richardson S. The timed "up \& go": A test of basic functional mobility for frail elderly persons. Journal of the American Geriatrics Society 1991;39:1428.
43. Schenkman M, Cutsom TM, Kuchibhatia $\mathrm{M}$, et al. Reliability of impairment and physical performance measures for persons with Parkinson's disease. Phys Ther 1997;77:19-27.

44. Visser M, Marinus J, Bloem B, et al. Clinical tests for the evaluation of postural instability in patients with Parkinson's disease. Arch Phys Med Rehabil 2003;84: 1169-74.

45. Pastor M, Day B, Marsden C. Vestibular induced postural responses in Parkinson's disease. Brain Injury 1993;5:1177-90.

46. Newton R. Review of tests of standing balance abilities. Brain Injury 1989;3:335-43.

47. Whitney S, Poole J, Cass S. A review of balance instruments for older adults. The American Journal of Occupational Therapy 1998;52:666-71.

48. Oxford Grice K, Vogel K, Le V, et al. Adult norms for a commercially available Nine Hole Peg Test for finger dexterity. American Journal of Occupational Therapy 2003;57:570-3.

49. Rosen C, Murry T. Voice Handicap Index in singers Journal of Voice 2000;14:370-7.

50. Turnbull J, Kersten P, Habib M, et al. Validation of the frenchay activities index in a general population aged 16 years and older Archives of Physical Medicine \& Rehabilitation 2000;81:1034-8.

51. Thornton M, Travis S. Analysis of the reliability of the modified caregiver strain index. Journal of Gerontology 2003;53B: S128-32.

52. Al-Janebi H, Coast J, Flynn T. What do people think when they provide unpaid care for an older person? A met-ethnography with interview follow-up. Social Science and Medicine 2008;67:111-21.

53. Horsman J, Furlong W, Feeny D, Torrance G. The Health Utilities Index (HUI): concepts, measurement, properties and applications. Health and Quality of Life Outcomes 2003;1:54. 